\title{
Using fault tree analysis to identify the failures of level crossing protection devices
}

\author{
J. C. Jong ${ }^{1}$, T. H. Lin ${ }^{1}$, C. S. Suen ${ }^{1}$, C. K. Lee ${ }^{2}$, \\ I. C. $\mathrm{Chen}^{3} \& \mathrm{H} . \mathrm{J} . \mathrm{Wu}^{3}$ \\ ${ }^{1}$ Civil \& Hydraulic Engineering Research Center, \\ Sinotech Engineering Consultants, Inc., Taiwan \\ ${ }^{2}$ Department of Marketing and Logistics, \\ Southern Taiwan University, Taiwan \\ ${ }^{3}$ Institute of Transportation, MOTC, Taiwan
}

\begin{abstract}
Level crossings are potential hazardous locations in a railway system. To reduce the risk of level crossings, several active protection devices such as flashing lights and barriers may be installed. Previous studies have proved that such protection devices are effective in decreasing the probability of accidents. However, these protection devices may be partially out of order or receive error signals. Once protection devices cannot operate correctly, they may deliver wrong information that confuses the users. As a result, the users may trespass a level crossing in a dangerous situation. To realize the causes of different types of wrong operations, this study categorized them into error warning, conflict warning, and no warning. Then fault tree analysis is employed to identify the failure types and the causes behind the wrong operations of protection devices, including the failures of flashing lights, boom barriers, train direction indicators, obstacle detectors, and emergency buttons. The results indicate that error warning is mostly due to the error messages of the track circuit. Conflict warning is caused by specific failures of the protection devices. Finally, the wiring error of the track circuit is the main cause leading to no warning.
\end{abstract}

Keywords: level crossing, protection device, fault tree analysis.

\section{Introduction}

In Taiwan, major cities are all developed along the conventional railroad that was original built at grade. Since the socio-economic activities on both sides of 
the railroad are quite close and active, the travel demand to cross the railroad is very high. Consequently, many level crossings exist along the railroad. Although some level crossings have been eliminated through elevated and underground railway projects, more than 500 level crossings are still distributed over the $1,097 \mathrm{~km}$ railroad. It means the average spacing between two crossings is about $2 \mathrm{~km}$. The high density level crossings and high traffic volume result in a high frequency of collision.

In addition to traffic signs and markings, many studies $[1,2]$ have proved that active protection devices could efficiently improve the safety of level crossings. For this reason, Taiwan Railway Administration (TRA), the operator of the conventional railroad, has gradually installed active protection devices in the last two decades. At present, most level crossings are mounted with flashing lights (which set up a chain reaction in audible devices), and more than 90 percent are equipped with boom barriers. Furthermore, TRA also installed emergency buttons on the roadsides of every level crossing and obstacle detectors at some dangerous sites.

Although the protection devices indeed decrease the frequency of collision, they create another safety problem. For example, in November 2009, the boom barriers of a level crossing did not descend while a train was approaching. Another event happened in January 2011. The boom barriers rose while the tail of the train still occupied the level crossing. Once the devices are out of order, roadside users (vehicle drivers or pedestrians) may receive wrong information and fall into the danger of collision. Besides, train drivers may also be confused by those incorrect operations of protection devices. To avoid these situations, all possible failures of protection devices and the causes behind these failures should be clearly clarified.

The safety issue of level crossings has continuously attracted considerable attention. For example, Pickett and Grayson [3] classified divers behaviour involving in accidents into three types: unwilling to stop, unable to stop, and unaware of the signal. Caird et al. [4] reviewed many studies and concluded four key factors causing collisions, including familiarity with the level crossing, traversing level crossings at slow speed, decision errors, and additional effects caused by active protection device. Siti [5] categorized the factors contributing to accidents at level crossing into human factor, engineering factor, and environmental factor. Although substantial studies have been devoted to the causes of collisions at level crossings, little research has considered the failures of protection devices.

The objective of this study is to clarify the relation between protection device failures and collisions. We started from examining the operating procedures and main components of five active protection devices used by TRA. Then the failures of protection devices which may lead to collisions were investigated. We found that five scenarios due to these failures could result in collisions. The fault tree diagrams were then employed to model the failure processes of the scenarios. The results of this study provide a framework to further study the risk of a level crossing and to develop strategies for improving safety. 


\section{The active protection devices}

After long-term development, most of TRA level crossings are currently equipped with five active protection devices, including boom barrier, flashing light, train direction indicator, emergency button, and obstacle detector. We reviewed Fu's [6] study and TRA rule books to illustrate the operating procedures and the components of these devices. In addition, we also referred to the reports of Proctor [7] to identify the failure types and the causes of protection devices.

\subsection{Boom barrier}

In the TRA system, almost all level crossings have four boom barriers to block the entrance and the exit of bi-directions. A few level crossings are installed with gate barriers, but they have been gradually removed or changed to boom barriers in recent years. Therefore, this section only discusses the operating procedure and possible failures of boom barriers.

\subsubsection{Operating procedure}

The boom barriers of a level crossing are interlocked with the track circuits on both ends of the level crossing. Fig. 1 shows the barriers start descending after 6-8 seconds while the upstream track circuit is occupied by an approaching train. It then takes 6-10 seconds to fully descend the barriers. The warning interval before the train arriving at the level crossing is at least 30 seconds. Sometimes, more than 2 trains may successively approach the level crossing. If the late coming train (either in the same or opposite directions) triggers the level crossing while the barriers are in the process of descending or already fully closed, the blocking time will extend until the late coming train leaves. However, if the level crossing is triggered while the barriers are in the rising procedure, the barriers continue rising until fully opened, and then descend immediately.

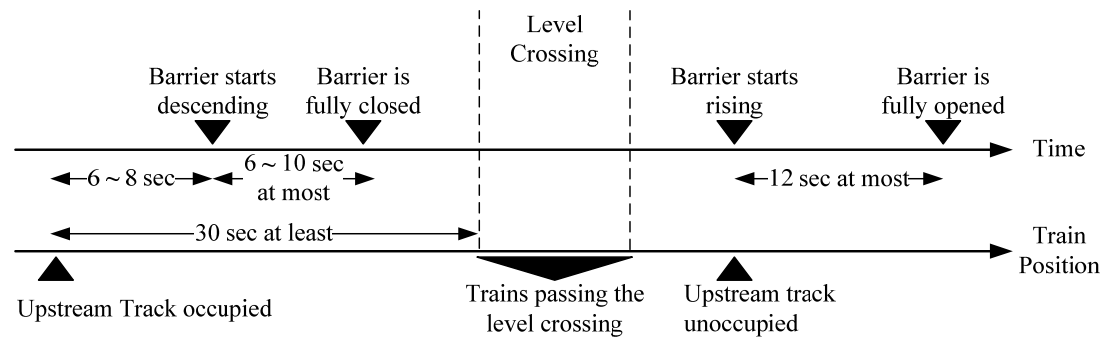

Figure 1: $\quad$ The operating procedure of boom barriers.

\subsubsection{Main components and failure types}

In addition to the barriers, level crossing is also composed of electric brake, gear motor and balance hammer. The electric brake is used to lock the barrier at the horizontal or vertical position. The gear motor can energise to rise or descend the 
barriers. The balance hammer makes barrier rise or descend more smoothly, and balances the torque when the barrier is at horizontal position. Due to fail-safe design, barrier should fall free to the horizontal position under failure conditions. However, if the hammer is blocked by obstacle, the fail-safe mechanism is broken.

\subsection{Flashing light and train direction indicator}

The flashing light system includes two red lamps which flash 30-40 times per minute. The train direction indicator is usually installed above the level crossing. Since the two devices have similar mechanism and always operate simultaneously, the study introduces them together.

\subsubsection{Operating procedure}

Fig. 2 shows an example of operating procedure of flashing lights and direction indicators. When the upstream track circuit of a level crossing is occupied, the flashing lights and the audible devices are triggered to operate immediately. At the same time, the direction indicator starts to function. Furthermore, the indicator can distinguish train direction and show the error message if warning time exceeds the maximum limit.

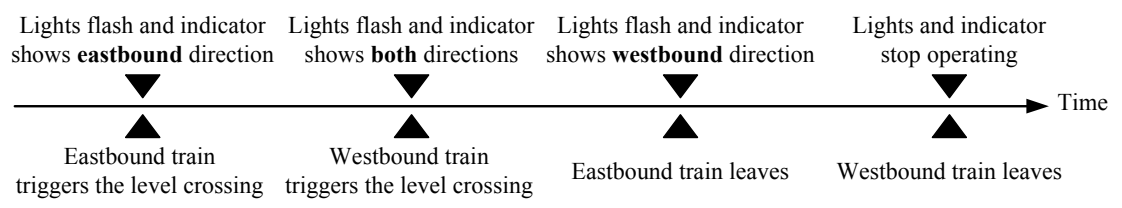

Figure 2: $\quad$ Operating procedure of flash lights and train direction indicators.

\subsubsection{Main components and failure types}

In TRA, typical incandescent bulbs are used for flashing lights and LED lamps are installed in train direction indicator. The incandescent bulbs may burn out, but the LED lamps would not completely fail unless losing power. Thus, device failures only happen in two situations, one is both incandescent bulbs of flashing lights burn out, and the other is losing power in both systems.

\subsection{Emergency button}

To provide train drivers with sufficient reaction time, TRA has installed emergency buttons on every level crossing.

\subsubsection{Operating procedure}

Fig. 3 shows the schematic diagram of emergency button system. Once the button is pushed, it will trigger three warning devices. One is the local broadcasting system. It will alarm vehicle drivers and pedestrians to leave crossing area as soon as possible. Another is trackside warning system, which consists of two pentagon warning lights located at 800 meters (far-end) and 200 500 meters (near-end) away from the level crossing, respectively. The other is 
the in-cab warning system. The radio waves emitted by the emergency buttons will notify all trains within a circle of two-kilometer radius centered at the level crossing. Once the train gets the emergency message, light and audible alarm will prompt in the cab.

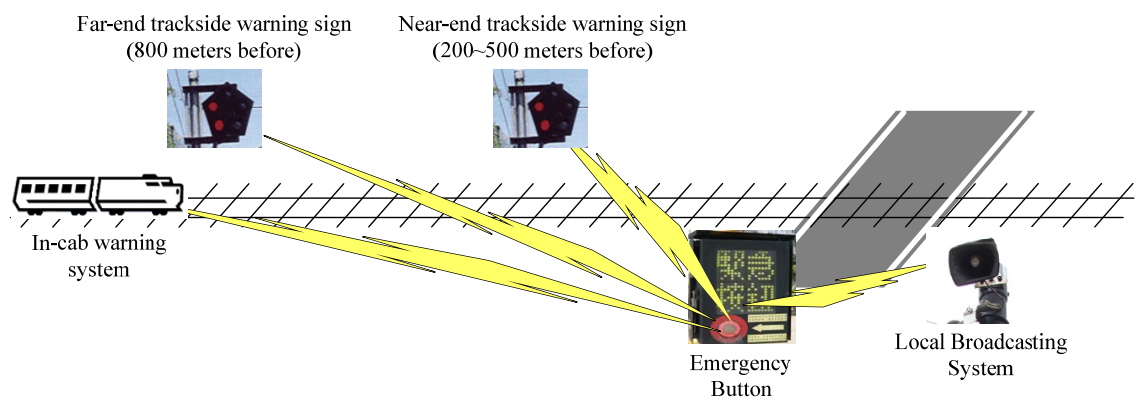

Figure 3: A schematic diagram of emergency button system.

\subsubsection{Main components and failure types}

Both wired and wireless transmissions are used by the emergency button system. Wired transmission is employed to transmit the signal to local broadcasting system and trackside warning system. Wireless transmission is used to trigger the in-cab warning system. Because the wires are exposed outside, they may be damaged by vandalism or external forces. On the other hand, the main threat to wireless is the communication interruption.

\subsection{Obstacle detector}

Fig. 4 shows a schematic diagram of obstacle detector system. The dotted lines represent the laser beams between the transmitters and receivers. Once one of the receivers is unable to detect the laser beam, it will transmit a warning signal to trackside warning system and trigger the pentagon warning lights.

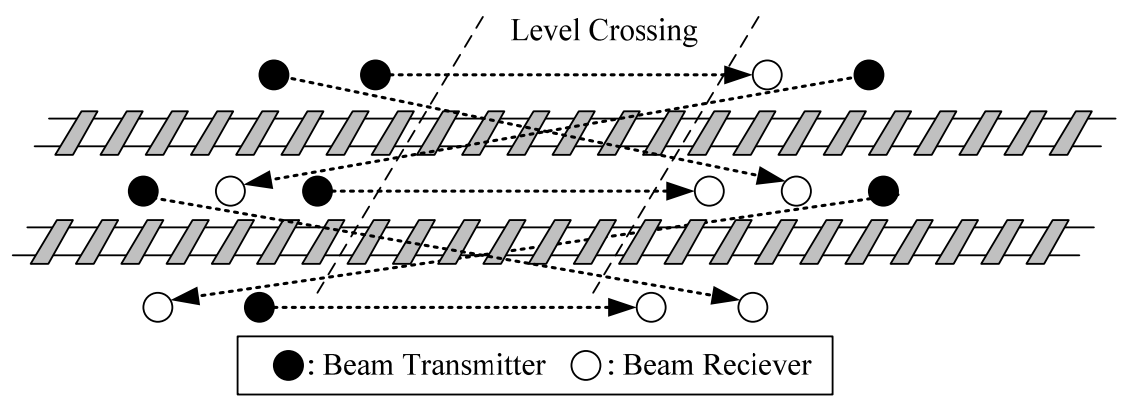

Figure 4: A schematic diagram of obstacle detection system. 


\subsubsection{Operating procedure}

When a train approaches a level crossing, each beam transmitter will shoot a laser ray to the corresponding receiver. If obstacles stay on the clearance area, the laser beams will be obstructed and the trackside warning signs will be triggered to alarm the train drivers.

\subsubsection{Main components and failure types}

Beam transmitters and receivers are main components in this system. The number of the detectors is determined according to the roadway length of the level crossing. Since the warning signal is only sent to trackside warning system, train drivers may miss the information if they have already passed the trackside warning signs. Besides, beam transmitters and receivers may be damaged by vandalism or external forces. Once transmitters or receivers fail, the system will suppose the laser beam is obstructed by the obstacle and start to warn.

\subsection{Summary}

In this section, we illustrate possible failures of each protection device. They are briefly listed in Table 1:

Table 1: $\quad$ Possible failures of protection devices.

\begin{tabular}{|c|c|c|}
\hline Device & Components & Failure Description \\
\hline \multirow{3}{*}{ Boom barriers } & Electric brake & $\begin{array}{l}\text { 1.Losing power } \\
\text { 2.Equipments fail to hold barriers }\end{array}$ \\
\hline & Gear motor & $\begin{array}{l}\text { 1.Losing power } \\
\text { 2.Equipments fail to raise or lower barriers }\end{array}$ \\
\hline & Balance hammer & 1.Be blocked \\
\hline Flashing lights & $\begin{array}{l}\text { Traditional incandescent } \\
\text { bulbs }\end{array}$ & $\begin{array}{l}\text { 1.Losing power } \\
\text { 2.Bulbs burn out }\end{array}$ \\
\hline $\begin{array}{l}\text { Train direction } \\
\text { Indicator }\end{array}$ & Led lamps & 1.Losing power \\
\hline \multirow{3}{*}{ Emergency button } & $\begin{array}{l}\text { Trackside warning sign } \\
\text { (far-end / near-end) }\end{array}$ & $\begin{array}{l}\text { 1.Losing power } \\
\text { 2. Wired break } \\
\text { 3.Bulbs burn out }\end{array}$ \\
\hline & In-cab warning system & $\begin{array}{l}\text { 1.Transmission interference } \\
\text { 2.Bulbs burn out } \\
\text { 3.Equipments fail to warn }\end{array}$ \\
\hline & Broadcasting system & $\begin{array}{l}\text { 1. Equipments fail to alarm } \\
\text { 2. Wired break }\end{array}$ \\
\hline \multirow{2}{*}{ Obstacle detector } & Beam transmitter & $\begin{array}{l}\text { 1.Equipments fail to transmit laser } \\
\text { 2.Damaged by external force }\end{array}$ \\
\hline & Beam receiver & $\begin{array}{l}\text { 1.Equipments fail to receive laser } \\
\text { 2.Damaged by external force }\end{array}$ \\
\hline
\end{tabular}

\section{The scenarios induced by error, conflict, and no warning}

The possible failures of protection devices are already introduced in the previous section. However, not all failures will lead to danger situations. Only when specific combinations of failures coincide with the presence of train will result in 
hazardous conditions. To clarify the situations, we have investigated TRA accident reports. We found that dangerous situations due to the failures of protection devices can be classified into error warning, conflict warning, and no warning of protection devices. Error warning means protection devices are in action while no train is present. Conflict warning means only part of devices are activated when a train is approaching. In other words, the protection devices deliver inconsistent message to users.

When taking train drivers and roadside users into account, the above three incorrect warnings can be further classified into five scenarios. The scenarios will be illustrated in the following subsections. Since track circuit plays an important role in activating protection devices, we also consider the possible failures of the track circuit (error signal) in the analysis.

\subsubsection{Scenario 1: dangers ahead - train drivers receive conflict warnings}

Two conditions which may confuse train drivers are discussed here. One is that when somebody pushes the emergency button, the in-cab warning system is activated, but either far-end or near-end trackside warning sign fails to function. The second condition may occur when obstacle detectors start to warn while the emergency button is not pushed. If the far-end trackside warning sign is in action but the near-end one fails, train drivers may suppose the obstacle has been cleared.

\subsubsection{Scenario 2: dangers ahead - train drivers do not receive any warnings}

This is a very dangerous situation. Two possible causes may lead to this circumstance. One is that somebody pushes the emergency button, but all devices are out of order. The other is that only obstacle detectors are triggered, but both the trackside warning signs fail to work.

\subsubsection{Scenario 3: no train approaching - roadside users receive error warnings}

If protection devices are triggered by error signal while no train is approaching, vehicle drivers or pedestrians may lose their patience and then start to trespass the level crossing after waiting for a long time. If a train is coming at this moment and roadside users are unaware of the train, collisions may happen. This is usually caused by error messages from track circuits. Moreover, if the electric brake is out of order or losing power, the barriers will be lowered down by the gravity even though no train is coming. Another situation is that barriers fail to rise after a train leaves. This may be caused by the blockage of the balanced hammer or the failure of the gear motor.

\subsubsection{Scenario 4: trains approaching - roadside users receive conflict warnings}

The scenario is resulted from the failures of part of protection devices. For example, if a train approaches while protection devices lose power, the barrier will fall down due to fail-safe mechanism. In this situation, flashing lights and train direction indicators would not work. Another situation is that the barrier is 
blocked in vertical position while a train is approaching. Roadside users may also get confused.

\subsubsection{Scenario 5: trains approaching - roadside users do not receive any warnings}

This is the most dangerous scenario and it indeed happens in TRA system. Two reasons may lead to this situation. One is that all protection devices are out of order and the barriers are blocked in vertical position. The other is error wiring of protection devices, which is also the major cause that happened in TRA.

\section{Modelling}

To clarify the causes leading to each scenario, we employed the Fault Tree Analysis (FTA) to model the failure modes of each scenario. FTA uses the logic gates to display the interrelation between the potential events (output) and the causes (input) to these events. Three kinds of gates are used in this study. The "And" gate indicates that output occurs only when all inputs occur. The "Or" gate means that output occurs if any input occurs. The "Inhibit" gate represents the output occurs if the inputs occur under an enabling condition specified by a conditioning event. In this study, we treated the above-mentioned five scenarios as the top events of the fault tree diagrams. Since the top events are safety hazards to either train drivers or roadside users their fault tree diagrams will be discussed separately.

\subsection{Safety hazards from the viewpoints of train drivers}

The fault tree for scenario 1 is displayed in Fig. 5 while that for scenario 2 is in Fig. 6. Both diagrams describe the situation while trains have not pass the farend trackside warning sign. From Fig. 5 and Fig. 6, we observe that the following mitigation measures could be used to reduce the risks of top events:

Near-end trackside warning sign should be more reliable than far-end one Wire should be protected and inspected periodically

Dual power loop and uninterruptible power supply (UPS) are necessary for redundancy;

LED is a good substitute for traditional incandescent bulbs.

\subsection{Safety hazards from the viewpoints of roadside users}

Figures 7 to 9 display the fault tree diagrams for scenarios 3-5, respectively. From Figs. 7 to 9 we observe the following phenomena:

Error track occupied signal directly results in error warnings.

It is efficient to reduce the frequency of conflict warning and no warning through the protection of balance hammer.

Error wiring will cause no warning while the local broadcasting system does not alarm.

Dual power loop or UPS is necessary.

Incandescent bulb is better to be replaced by LED. 


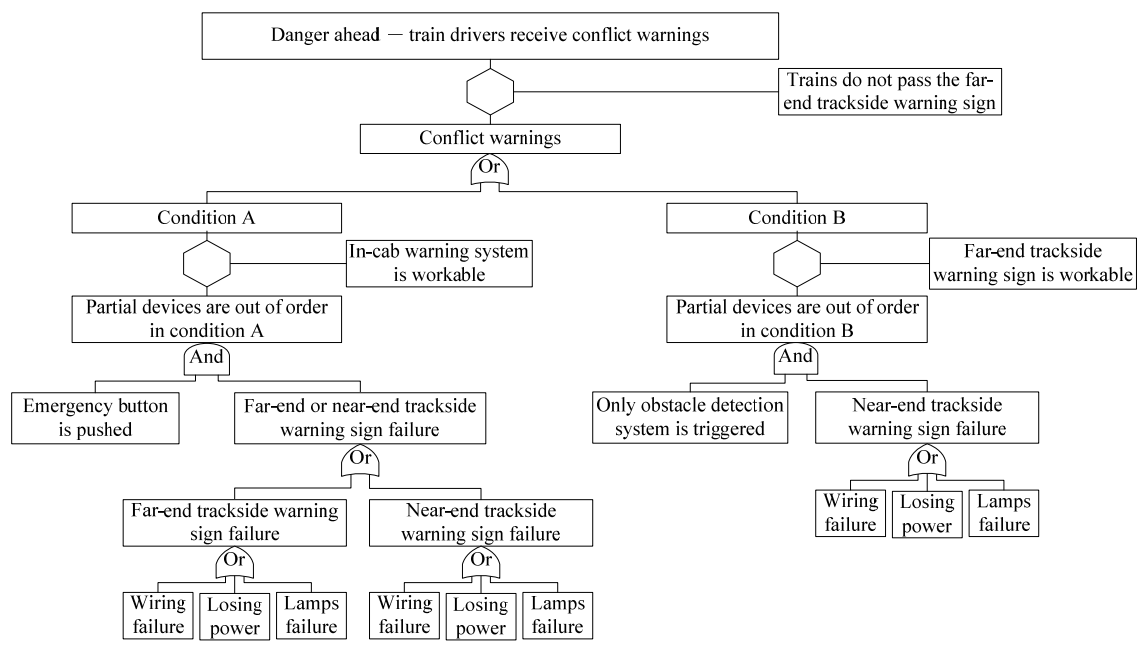

\section{Figure 5: The fault tree diagram of scenario 1.}

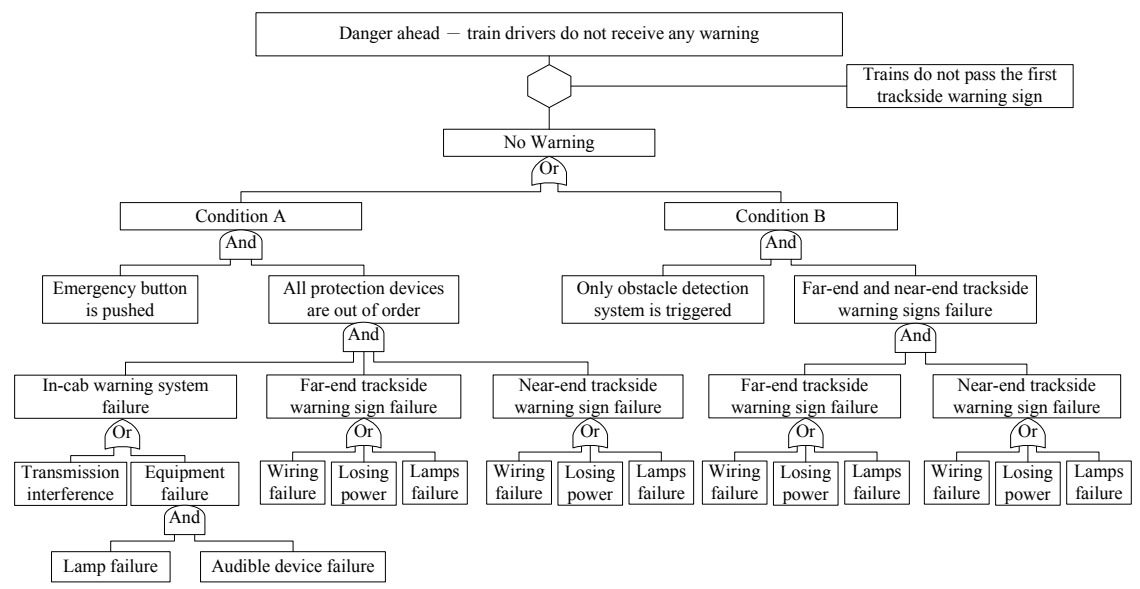

Figure 6: The fault tree diagram of scenario 2 . 


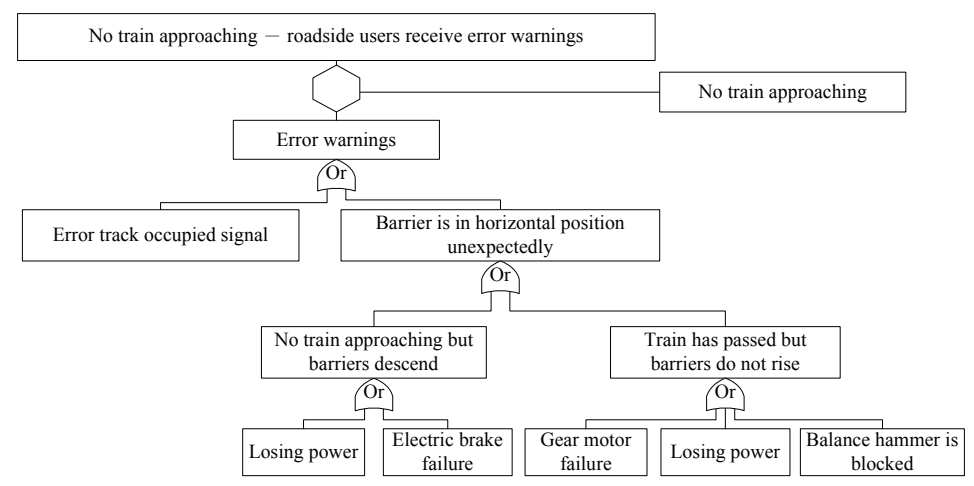

Figure 7: The fault tree diagram of scenario 3.

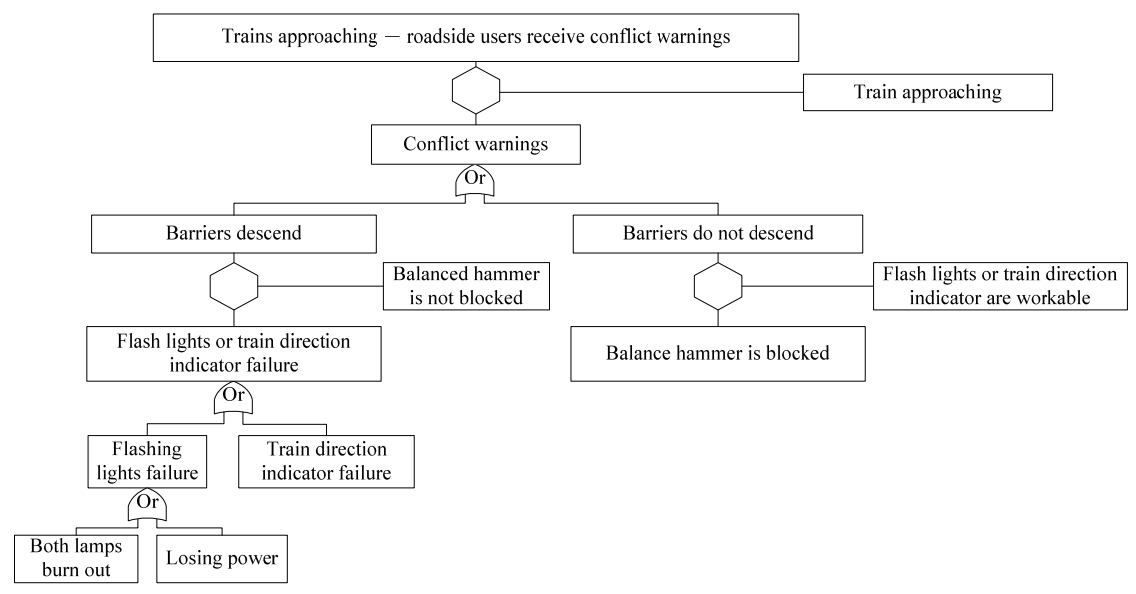

Figure 8: $\quad$ The fault tree diagram of scenario 4 . 


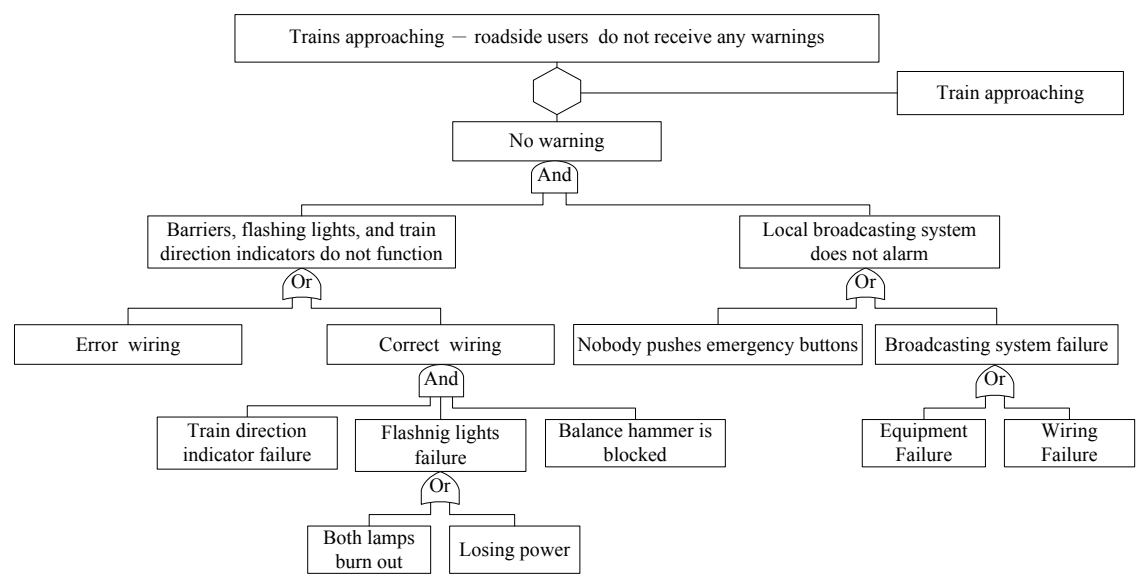

Figure 9: The fault tree diagram of scenario 5.

\section{Conclusion and suggestion}

The study took TRA system for example and discussed the active protection devices of level crossing. The failure reports of TRA were analyzed and five device failure scenarios which may lead to the collision were concluded. It is believed that the collisions could be prevented if the protection devices function correctly and road users do not violate traffic rules. However, the protection devices may fail to work, deliver error messages, or conflict messages. The unexpected events will confuse both train drivers and roadside users, and then trap them into dangerous situations. It is recommended that railway operators should focus on the key failures leading to these five scenarios. For example, the near-end trackside warning sign should have higher reliability than the far-end one. Losing power must be avoided by back-up power supply. Using LED is more reliable than traditional incandescent bulbs. Finally, railway operators could use the fault tree diagrams developed in this study to evaluate the investment benefit of protection devices.

\section{Reference}

[1] Fambro, D. B. et al., Evaluation of two active traffic control devices for use at railroad-highway grade crossings, Transportation Research Record, 1244, pp. 52-62, 1989.

[2] Wigglesworth, E. C. and Uber, C. B., An evaluation of the railway level crossing boom barrier program in Victoria, Australia, Journal of Safety Research, 22, pp. 133-140, 1991.

[3] Pickett, M.W., Grayson, G.B., Vehicle Driver Behaviour at Level Crossings, Transport Research Laboratory, UK, 1996. 
[4] Caird, J.K., Creaser, J.I., Edwards, C.J. \& Dewar, R.E., A Human Factors Analysis of Highway-Railway Grade Crossing Accidents in Canada, Transport Canada, Transportation Development Centre, 2002.

[5] Siti, Z.I., The development of railway level crossing safety assessment model: a research framework. $29^{\text {th }}$ Conference of Australian Institutes of transport Research, 2007.

[6] Fu, Y.H., Application remote video monitoring for improvement of railway crossing safety, master thesis of National Taipei University of Technology, 2007.[in Chinese: English Abstract].

[7] Proctor, P., Infrastructure Risk Modelling: Automatic Level Crossing, Automatic Half Barrier Type, Railtrack EE\&CS, 1997. 